\title{
Válvula aórtica cuadricúspide: caso clínico y discusión
}

Julián Sepúlveda ${ }^{1}$, José Torres ${ }^{1}$, Leonel López ${ }^{2}$, Hernán Donoso ${ }^{2}$, Jaime Micolich².

1 Residente Cardiología, Universidad de Chile.

2 Departamento de Cardiología, Hospital San Juan de Dios, Santiago de Chile.

\section{Quadricuspid aortic valve}

The quadricuspid aortic valve is a rare congenital anomaly, usually presenting as an isolated malformation causing aortic regurgitation in the faith or sixth decades of life. The first reported cases were found at autopsy or became evident by surgical findings. However, the emergence of new imaging modalities currently allows an earlier diagnosis, including in asymptomatic pa- tients. Hereby the case of a 60 -year-old woman with quadricuspid aortic valve diagnosed by echocardiography is presented. A brief discussion of the disease is included

Keywords: quadricuspid aortic valve; congenital heart disease; aortic regurgitation; echocardiography. 


\section{Introducción:}

La válvula aórtica cuadricúspide (VAC) es una rara anomalía congénita, con una incidencia reportada del 0.008 y $0.043 \%$ en estudios de autopsia y ecocardiografía respectivamente. Es menos frecuente en comparación con válvula aórtica bicúspide $(1-2 \%)$ o unicúspide $(<1 \%)^{1-2}$. Durante su evolución aparece frecuentemente regurgitación aórtica, a menudo pura, entre la quinta y sexta décadas de la vida ${ }^{4-5}$.

\section{Caso clínico:}

Mujer de 60 años, con antecedentes de artritis reumatoide inactiva de 20 años de evolución, en tratamiento con lefunomide y metotrexato.

Derivada desde Reumatología por hallazgo de soplo cardíaco. La paciente era asintomática, con capacidad funcional I. La presión arterial era 140/80 mmHg, frecuencia cardíaca $74 \mathrm{lpm}$ regular y presentaba un escape aórtico grado $3 / 6$ en $2^{\circ}$ espacio intercostal para esternal derecho. No tenía signos de insuficiencia cardíaca.

El electrocardiograma mostró ritmo sinusal, frecuencia cardíaca de 76 lpm, sin alteraciones de la conducción ni signos de hipertrofia ventricular. La radiografía de tórax era normal.

En la ecocardiografía transtorácica se observó cavidades de tamaño conservado, sin trastornos de la motilidad global ni segmentaria. La válvula aórtica mostraba un déficit de coaptación con una regurgitación de grado moderado (Vena Contrasta de $0.5 \mathrm{~cm}$ y Doppler continuo con tiempo desaceleración de $374 \mathrm{mseg}$, (Figura 1).

Para definir mejor la morfología valvular se realizó una ecocardiografía transesofágica, donde se aprecia imagen de cuatro cúspides aórticas, con similar tamaño por planimetría, Se apreciaba un déficit de coaptación que provocaba regurgitación moderada con un chorro central al doppler color. No existían otras alteraciones estructurales (Figura 2).

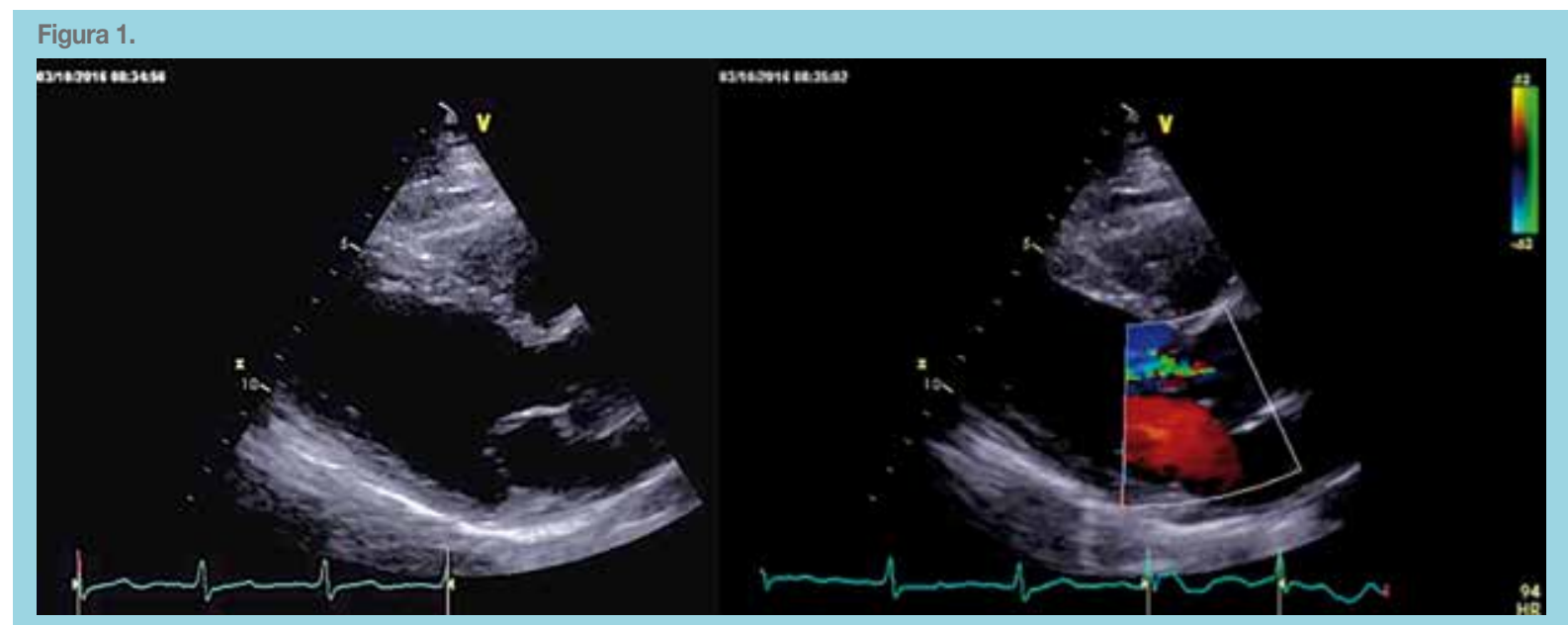

Ecocardiografía TT, a la izquierda eje para esternal largo con cavidades de tamaño conservado, a la derecha doppler color sobre la válvula aórtica.

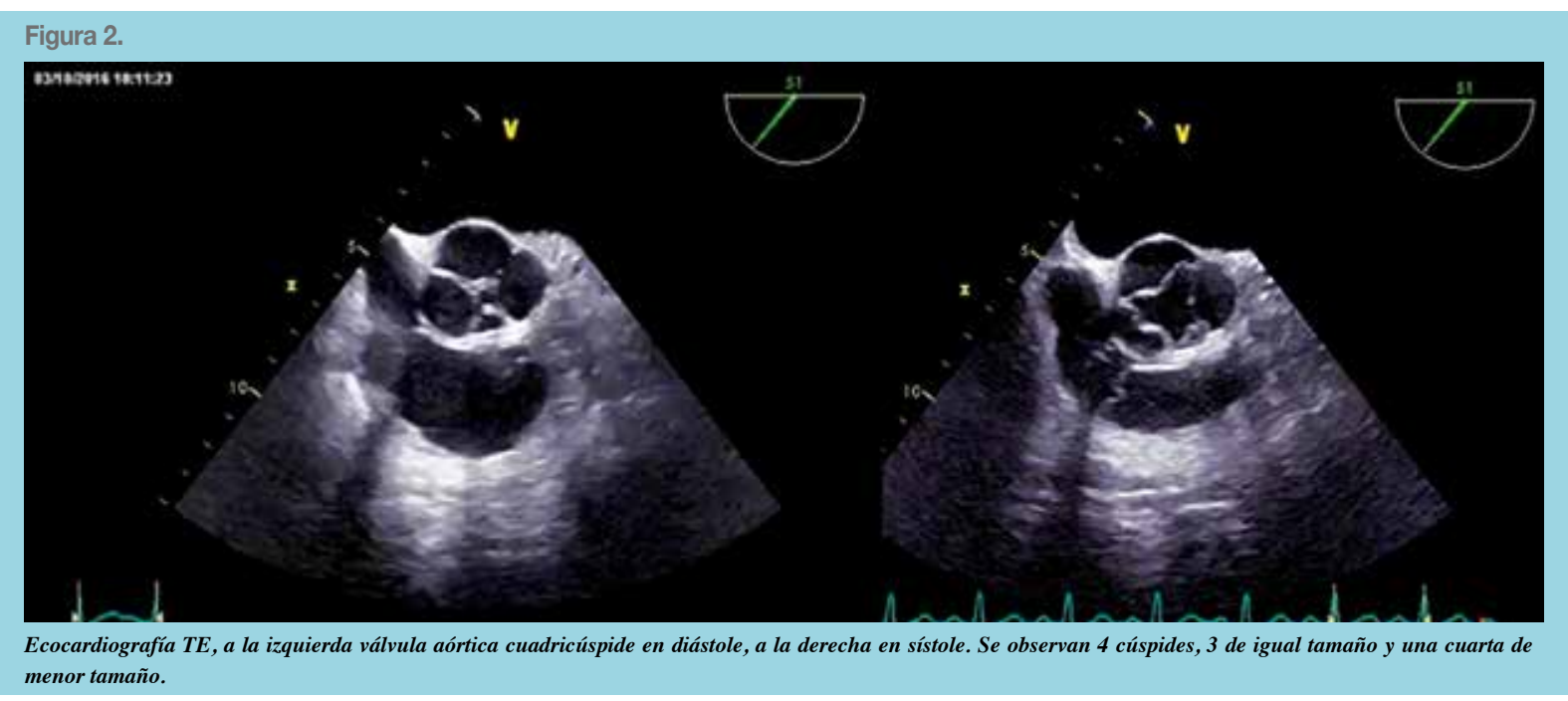




\section{Discusión}

La válvula aórtica cuadricúspide es una anomalía congénita poco frecuente ${ }^{1-2}$. El primer caso fue descrito por Balington en 1862, tras estudios en autopsias. La incidencia real es difícil de estimar, ya que en general constituye un hallazgo posquirúrgico o por estudio de imágenes1-2. Tutarel en 2004 publicó una incidencia basada en estudios de autopsia combinada con ecocardiografía del $0.01 \%{ }^{3}$. Una reciente revisión reporta una incidencia del $0.043 \%$ en estudios de ecocardiografía y de $0.05 \%$ tras el reemplazo valvular ${ }^{8}$. Con el advenimiento de la ecocardiografía y otras técnicas de imágenes, se han informado cada vez más casos de VAC.

Existen diversas hipótesis embriológicas para explicar esta anomalía. Se ha sugerido la tabicación anormal del tronco común (conotruncus) y la tabicación anormal de uno de los cojines endocardios tras un episodio inflamatorio ${ }^{4-5}$. Aparece habitualmente como anomalía congénita aislada. El desarrollo de las valvas aórticas se produce después del proceso de formación de las arterias coronarias en los senos de Valsalva, por lo cual se le relaciona con el origen anómalo de las arterias coronarias $^{4-5}$. Otras anomalías cardíacas descritas con mayor frecuencia son defecto septal ventricular, cardiomiopatía hipertrófica no obstructiva, estenosis de la válvula pulmonar, ductus arterioso persistente, estenosis subaórtica y supra valvular ${ }^{3-4-5}$.

Se han descrito diferentes variantes anatómicas. Hurwitz y Roberts, en 1973, las clasifican de acuerdo con el tamaño y simetría de las cúspides, en siete grupos, clasificación que continua vigente ${ }^{9}$. El tipo A presenta cuatro cúspides iguales; el $\mathrm{B}$ tres cúspides iguales y una más pequeña; el C, dos cúspides grandes iguales y dos pequeñas iguales; el D, una grande, dos intermedias y una pequeña cúspide; el E, tres cúspides iguales y una grande; el F, dos cúspides grandes iguales y dos pequeñas desiguales; y el tipo G, cuatro cúspides desiguales (Figura 3$)^{9}$.

Nakamura et al. ${ }^{10}$ diseñó una clasificación simplificada, tras revisión en 42 pacientes, Se basa en la posición de la cúspide supernumeraria: tipo I, cúspide supernumeraria entre las cúspides coronarias izquierda y derecha; tipo II, cúspide supernumeraria entre las cúspides derecha y no coronaria; tipo III, cúspide supernumeraria entre las cúspides izquierda y no coronarias; y el tipo IV, cúspide supernumeraria no identificada entre dos cúspides pequeñas de igual tamaño. La frecuencia de los cuatro tipos es $24 \%, 31 \%, 7 \%$ y $5 \%$ respectivamente ${ }^{9-10}$. Los tipos I y II de la clasificación simplificada son similares a los tipos A y B de Hurwitz y Roberts.
Hasta el momento, se han publicado pocas series y muchos casos aislados, y los tipos A, B y C de Hurwitz et al representan más del $85 \%$ de los casos. Nuestra paciente corresponde al Tipo B (3 velos igual y uno menor), descrita como la forma más común.

Se desconoce la relación entre la clasificación morfológica y la evolución de la anomalía ${ }^{4}$. Sin embargo, se estima que, a mayor desigualdad en tamaño y simetría de las valvas, estos experimentan mayor tensión, alteración en la coaptación y se facilita a largo plazo una regurgitación aórtica significativa ${ }^{4-5-6}$.

Figura 3. Válvula Cuadricúspide, clasificación de 7 subgrupos, adaptación de acuerdo a Hurwitz y Roberts?

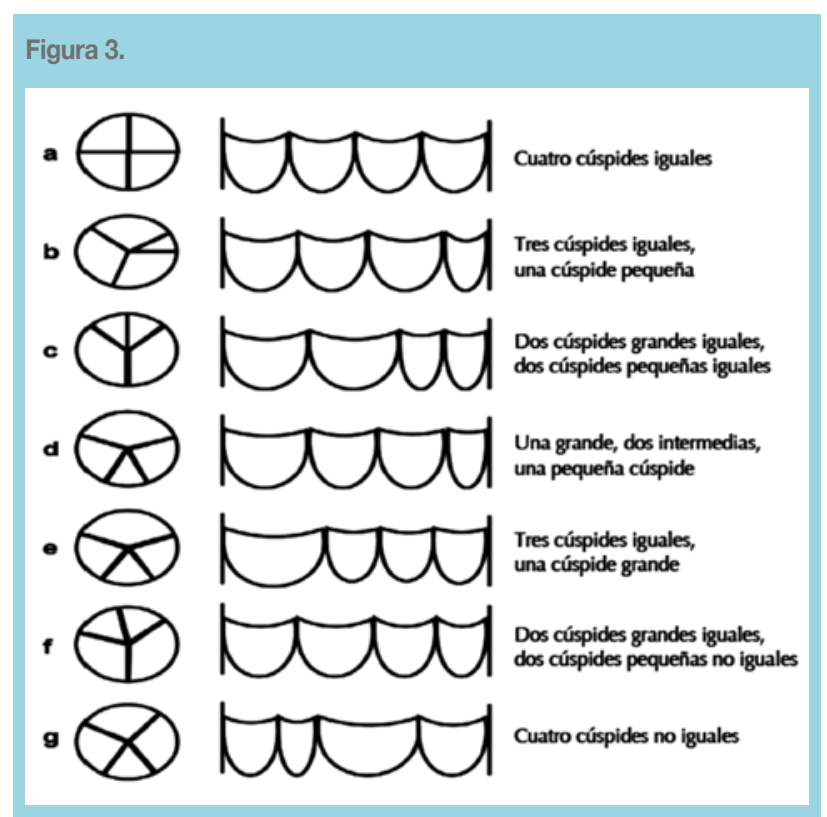

Las manifestaciones clínicas de pacientes con VAC dependen del estado funcional de la anomalía y los trastornos asociados. En general los pacientes son asintomáticos hasta la sexta década de la vida ${ }^{4-8}$.

La ecocardiografía transtorácica bidimensional permite detectar anomalías estructurales, pero es frecuente que se requiera ecocardiografía transesofágica que proporciona imágenes más fiables y permite precisar más un mayor número de casos de $\mathrm{VAC}^{4-8}$. Las anomalías del ostium coronario son los trastornos asociados más frecuentes, hasta $10 \%$ de los casos de VAC ${ }^{6-7}$. Por lo tanto, la ecocardiografía transesofágica es la herramienta de elección para definir la morfología valvular y estas lesiones asociadas $^{6-8}$.

Importante es distinguir la VAC verdadera producto de la embriogénesis anormal, de las pseudo VACs, resul- 
tantes de secuelas de la endocarditis infecciosa o fiebre reumática 4 .

La tomografía computarizada cardíaca y la resonancia magnética tienen un alto valor diagnóstico, pero no se aconseja su uso diagnóstico en la práctica clínica. ${ }^{4-8}$

Esta anomalía se asocia a regurgitación aórtica en más del $75 \%$ de los casos. En un estudio la función valvular fue normal en $16 \%$ de los casos, la regurgitación asociada a estenosis en $8,4 \%$, mientras que la estenosis aórtica pura lo fue en $0.7 \%{ }^{4-6}$. La mayor progresión a regurgitación moderada-severa se presenta entre la quinta y sexta década ${ }^{4-6}$. La distribución desigual de tensión y la coaptación incompleta de las cúspides determina un mayor riesgo de progresión de la regurgitación ${ }^{8}$. A diferencia de la válvula aórtica bicúspide, la VAC no está asociada a dilatación de la aorta ascendente. De hecho, la mayor parte de los casos presentan la raíz aórtica y la aorta ascendente de tamaño normal ${ }^{8}$.

La endocarditis infecciosa se presenta en el 1,4\% de los casos. Se considera la cúspide supernumeraria, la alteración geométrica, la desigual distribución de tensión y el defecto de coaptación como factores de riesgo de $\mathrm{EI}^{5-8}$.
No hay acuerdo respecto a la profilaxis de la endocarditis infecciosa en pacientes con VAC. Algún autor recomienda la profilaxis con antibióticos en todos los $\operatorname{casos}^{11} \mathrm{y}$ otros solo en casos de dismetría valvular. Sin embargo, las directrices de la ESC y la ACC/AHA 2015 no recomienda la profilaxis con antibióticos sin evidencia de otra infección activa ${ }^{12}$.

Las indicaciones quirúrgicas para VAC, son la regurgitación aórtica severa, la estenosis aórtica severa o VAC disfuncional asociada con otras lesiones, como la oclusión del ostium coronario izquierdo ${ }^{6-8}$. El reemplazo valvular es la técnica quirúrgica de elección; sin embargo, en casos seleccionados se efectúa la reparación valvular. La técnica de reparación más común es la tricuspidización de la válvula aórtica, usando tejido de pericardio autólogo o de bovino. El objetivo de la reparación es restaurar una coaptación precisa, de bajo gradiente transvalvular, sin flujo turbulento y lograr mayor durabilidad a largo plazo $^{13}$. Sin embargo, los casos son limitados y con escasos seguimiento. Cabe mencionar que el reemplazo valvular transcatéter no se recomienda para los pacientes con VAC y regurgitación ${ }^{4-13-14}$. 


\section{Referencias}

1. FELDMAN BJ, KHANDERIA BK, WARNE CA, SEWARD JB, TAYLOR CI, TAJIK AJ. Incidence, description and function assessment of isolated quadricuspid aortic valves. Am J Cardiol 1990; 65: 937- 8 .

2. TIMPERLEY J, MILNER R, MARSHALL AJ, GILBERT TJ. Quadricuspid aortic valves. Clin Cardiol 2002; 25: 548-52.

3. TUTAREL O. The cuadriscuspid aortic valve: A comprehensive review. J Heart Valve Dis 2004, 13: 534-37.

4. SAVINO K, QUINTAVALLE E, AMBROSIO G. Quadricuspid aortic valve: A case report and review of the literature. $J$ Cardiovasc Echogr. 2015; 25: 72-6.

5. YUAN S-M. Quadricuspid Aortic Valve: A Comprehensive Review. Brazilian J Cardiovasc Surg. 2016; 3: 454-60.

6. ZHU J, ZHANG J, WU S, ZHANG Y, DING F, MEI J. Congenital quadricuspid aortic valve associated with aortic insufficiency and mitral regurgitation. J Cardiothorac Surg 2013; 8: 87.

7. TIMPERLEY J, MILNER R, MARSHALL AJ, GILBERT TJ Quadricuspid aortic valves. Clin Cardiol 2002; 25: 548-52.

8. TSANG MY, ABUDIAB MM, AMMASH NM, NAQVI TZ, EDWARDS WD, NKOMO VT, et al. Quadricuspid aortic valve: Characteristics, associated structural cardiovascular abnormalities, and clinical outcomes. Circulation 2016; 133: $312-9$.
9. HURWITZ LE, ROBERTS WC. Quadricuspid semilunar valve. Am J Cardiol 1973; 31: 623-6.

10. NAKAMURA Y, TANIGUCHI I, SAIKI M, MORIMOTO K, YAMAGA T. Quadricuspid aortic valve associated with aortic stenosis and regurgitation. Jpn J Thorac Cardiovasc Surg. 2001; 49: 714-6.

11. BAUER F, LITZLER PY, TABLEY A, CRIBIER A, BESSOU JP. Endocarditis complicating a congenital quadricuspid aortic valve. Eur J Echocardiogr. 2008; 9: 386-7.

12. HABIB G, LANCELLOTTI P, ANTUNES MJ, BONGIORNI MG, CASALTA JP, DEL ZOTTI F, et al. 2015 ESC Guidelines for the management of infective endocarditis: The Task Force for the Management of Infective Endocarditis of the European Society of Cardiology (ESC) Endorsed by: European Association for Cardio-Thoracic Surgery (EACTS), the European Association of Nuclear Medicine (EANM). Eur Heart J. 2015; 36: 3075-128.

13. SONG MG, YANG HS, LEE DH, SHIN JK, CHEE HK, KIM JS. Mid-term results in patients having tricuspidization of the quadricuspid aortic valve. J Cardiothorac Surg. 2014; 9: 29

14. IDREES JJ, ROSELLI EE, ARAFAT A, JOHNSTON DR, SVENSSON LG, SABIK JF 3RD, et al. Outcomes after repair or replacement of dysfunctional quadricuspid aortic valve. J Thorac Cardiovasc Surg 2015; 150: 79-82. 